\title{
High-pT hadrons in heavy ion collisions: from RHIC to LHC
}

\author{
Jan Nemchik* \\ Czech Technical University in Prague, FNSPE, Břehová 7, 11519 Prague, Czech Republic; and \\ Institute of Experimental Physics SAS, Watsonova 47, 04001 Košice, Slovakia \\ E-mail: nemcikesaske.sk
}

\section{Boris Z. Kopeliovich}

Departamento de Física, Universidad Técnica Federico Santa María; and Instituto de Estudios Avanzados en Ciencias e Ingeniería; and

Centro Científico-Tecnológico de Valparaíso;

Casilla 110-V, Valparaíso, Chile

E-mail: boris.kopeliovich@usm.cl

\section{Irina K. Potashnikova}

Departamento de Física, Universidad Técnica Federico Santa María; and Instituto de Estudios Avanzados en Ciencias e Ingeniería; and

Centro Científico-Tecnológico de Valparaíso;

Casilla 110-V, Valparaíso, Chile

E-mail: irina.potashnikova@usm.cl

The length scale $l_{p}$ for production of leading hadrons in high- $p_{T}$ jets, constrained by energy conservation, is rather short and does not rise with $p_{T}$. Therefore, the dominant source of suppression of high- $p_{T}$ single hadrons is attenuation of the produced colorless dipoles in the dense matter created in heavy ion collisions, rather than the induced energy loss at the short early stage of hadronization. Since the evolution of the initially small dipole size up to the hadron dimension is slowed down by Lorentz time dilation, the dipole survival probability rises with $p_{T}$ in a good accord with LHC data. The $p_{T}$ dependence of $R_{A A}$ at the energies of RHIC is weaker due to closeness of the kinematic bound. This description is parameter free, except the absorption rate of the medium, which is the goal of this analysis and is adjusted to data. The maximal value of the coordinate- and time-dependent transport coefficient $\hat{q}$ is found to be $\hat{q}_{0}=0.30-0.45 \mathrm{GeV}^{2} / \mathrm{fm}$ at $\sqrt{s}=62-200 \mathrm{GeV}$; and $\hat{q}_{0}=0.60 \mathrm{GeV}^{2} / \mathrm{fm}$ at $\sqrt{s}=2.76 \mathrm{TeV}$. The impact parameter dependence of $R_{A A}\left(p_{T}\right)$ at different collision energies is successfully reproduced without further adjustments.

Sixth International Conference on Quarks and Nuclear Physics

April 16-20, 2012

Ecole Polytechnique, Palaiseau, Paris

\footnotetext{
* Speaker.
} 


\section{INTRODUCTION}

The last data on high- $p_{T}$ charged hadron production [1], 2] in heavy ion collisions (HIC) at LHC clearly demonstrate a strong nuclear suppression and discover also two novel features compared with PHENIX [3, 4] and STAR [5] measurements at RHIC. First, the nuclear modification factor $R_{A A}$ measured at LHC reaches significantly smaller values. Second, $R_{A A}\left(p_{T}\right)$ steeply rises with $p_{T}$ at LHC but exhibits a rather flat $p_{T}$ dependence at RHIC.

The explanation of observed high- $p_{T}$ suppression is connected with understanding of a hadronization mechanism, namely an energy loss scenario of a created parton after HIC. There are two time scales controlling the hadronization process related to production of a colorless dipole (pre-hadron) and the final hadron [6, 7]. The former results in color neutralization stopping the intensive energy loss, while the latter lasts much longer up to formation of the hadron wave function.

Here we rely on the model [8] for the production time $l_{p}$ distribution of leading hadrons in a jet produced at the mid rapidity. In this case the initial parton energy and virtuality are equal, $E=Q=k_{T}=p_{T} / z_{h}$, where $z_{h}$ is a fraction of the jet momentum carried by the hadron, $k_{T}$ and $p_{T}$ are the transverse momenta of the parton initiating the jet and of the detected hadron, respectively. The left panel of Fig. 1 1 shows the $p_{T}$ dependence of the mean $\left\langle l_{p}\right\rangle$ for quark (upper three curves) and gluon (lower three curves) jets at fixed $z_{h}=0.5,0.7,0.9$.
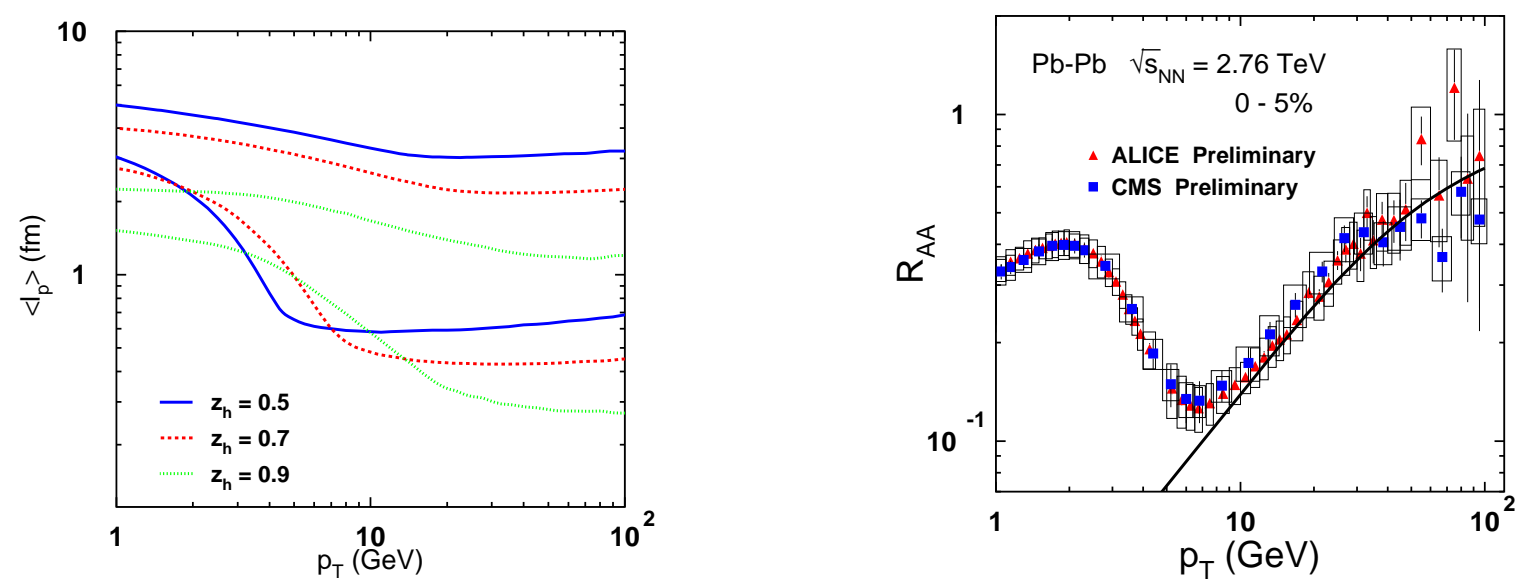

Figure 1: (Left) The mean production length as function of $p_{T}$ for quark (upper three curves) and gluon (lower three curves) jets at $z_{h}=0.5,0.7,0.9$ (from top to bottom). (Right) ALICE [1] and CMS 胞] data for charged hadrons produced in central, $0-5 \%$, lead-lead collisions at $\sqrt{s}=2.76 \mathrm{TeV}$ vs. calculations at adjusted value of $\hat{q}_{0}=0.6 \mathrm{GeV}^{2} / \mathrm{fm}$.

Note, that for gluon jets the energy loss is larger and the Sudakov suppression stronger due to the Casimir factor. This leads to a shorter production length corresponding to $\left\langle l_{p}\right\rangle<1 \mathrm{fm}$ at large $p_{T}$ as is demonstrated in the left panel of Fig. 1.

The recent study of $J / \Psi$ suppression [9] found that the density of the medium is rather low corresponding to the transport coefficient $\hat{q}_{0} \sim 0.2 \mathrm{GeV}^{2} / \mathrm{fm}$. Similar value $\hat{q}_{0} \sim 0.4 \mathrm{GeV}^{2} / \mathrm{fm}$ was found in [10] studying suppression of high- $p_{T}$ hadrons at LHC in central lead-lead collisions.

Here we employ the same description of dipole propagation in a medium as was used for hadron suppression at LHC [10] extending it also for non-central $A+B$ collisions and for lower energies. For a relative quark-gluon jet contributions to final hadron production we use the standard convolution expression based on QCD factorization [11]. For the RHIC energy range we include also the additional suppression factor related to the energy conservation constraints [12, 13]. 


\section{DIPOLE ATTENUATION IN A MEDIUM}

Contribution of the dipole attenuation to nuclear suppression is related to properties of the created medium described in terms of the transport coefficient $\hat{q}$. Here we rely on the usual assumptions that the initial medium density at time $t=t_{0}$ is proportional to the number of participants $n_{\text {part }}$, and the density is diluting with time as $1 / t$. Then the transport coefficient reads [14,

$$
\hat{q}(t, \vec{b}, \vec{\tau})=\frac{\hat{q}_{0} t_{0}}{t} \frac{n_{\text {part }}(\vec{b}, \vec{\tau})}{n_{\text {part }}(0,0)}
$$

where the parameter $\hat{q}_{0}$ represents the maximal value of $\hat{q}$, for the medium produced at $t=t_{0}$ in a central collision at $b=\tau=0$. Variables $\vec{b}$ and $\vec{\tau}$ are the impact parameters of collision and position of the parton. In what follows we treat the transport coefficient $\hat{q}_{0}$ as an adjusted parameter.

A small size dipole propagating in a medium attenuates with the cross section proportional to $r_{T}^{2}(t)$, where its transverse expansion reads [ㄱ. 10],

$$
r_{T}^{2}(t)=\frac{2 t}{\alpha(1-\alpha) \tilde{E}}+r_{0}^{2},
$$

where $\alpha$ is the fractional light-cone momentum of the parton, $\tilde{E}=p_{T}$ is the dipole energy in the c.m. of the collision and $r_{0} \sim 1 / p_{T}$ is the initial dipole size, neglected in what follows.

The nuclear attenuation factor at given impact parameter $b$ reads,

$$
R_{A B}\left(b, p_{T}\right)=\frac{\sigma_{A B}\left(b, p_{T}\right)}{\int_{0}^{\infty} d^{2} \tau T_{A}(\tau) T_{B}(\vec{b}-\vec{\tau}) \sigma_{p p}\left(p_{T}\right)},
$$

where cross sections $\sigma_{p p}\left(p_{T}\right)$ of the process $p+p \rightarrow h+X$ and $\sigma_{A B}\left(b, p_{T}\right)$ of the process $A+B \rightarrow$ $h+X$ are calculated using the standard convolution expression [11],

$$
\begin{gathered}
\sigma_{p p}\left(p_{T}\right)=\sum_{i, j, k, l} F_{i / p} \otimes F_{j / p} \otimes \hat{\sigma}_{i j \rightarrow k l} \otimes D_{h / k} \\
\sigma_{A B}\left(b, p_{T}\right)=\int_{0}^{\infty} d^{2} \tau T_{A}(\tau) T_{B}(\vec{b}-\vec{\tau}) \sum_{i, j, k, l} F_{i / A} \otimes F_{j / B} \otimes \hat{\sigma}_{i j \rightarrow k l} \otimes D_{h / k} R_{A B}^{k}\left(\vec{b}, \vec{\tau}, p_{T}\right) .
\end{gathered}
$$

Functions $F_{i / p}, F_{j / p}$ and $F_{i / A}, F_{j / B}$ in Eqs. (2.4) and (2.5) represent the distributions of parton species $i, j$ in Bjorken $x_{1}, x_{2}$ and transverse momenta in colliding protons and nuclei, respectively. For the parton distribution function in a nucleon we use the leading order MSTW-2008 parametrization [18]. The nuclear parton distributions, $F_{i / A}, F_{j / B}$ are parametrized according to [19]. For the hard parton scattering cross section $\hat{\sigma}_{i j \rightarrow k l}$ in Eqs. (2.4) and 2.5) we use regularization masses $m_{g}=0.8 \mathrm{GeV}$ and $m_{q}=0.2 \mathrm{GeV}$ for gluon and quark propagators, respectively. The fragmentation functions of a parton $k$ into the final hadron $h, D_{h / k}$ are taken from [20] in leading order. In Eq. (2.5) $T_{A}(b)=\int_{-\infty}^{\infty} \rho_{A}(b, z) d z$ is the nuclear thickness function, where for the nuclear density $\rho_{A}(b, z)$ we use the realistic Woods-Saxon paramerization [21].

The suppression factor $R_{A B}^{k}\left(\vec{b}, \vec{\tau}, p_{T}\right)$ in Eq. (2.5) has the form [ [7],

$$
R_{A B}^{k}\left(\vec{b}, \vec{\tau}, p_{T}\right)=\int_{0}^{\pi} \frac{d \phi}{\pi} \exp \left[-\frac{1}{\alpha(1-\alpha) p_{T}} \int_{l_{\max }^{k}}^{\infty} d l l \hat{q}(l, \vec{b}, \vec{\tau}+\vec{l})\right],
$$

where $l_{\max }^{k}=\max \left\{\left\langle l_{p}^{k}\right\rangle, l_{0}\right\}$. Here $t_{0}=l_{0} \sim 1 \mathrm{fm}$ is the time scale of the medium creation. 


\section{ENERGY CONSERVATION CONSTRAINTS}

In the RHIC kinematic region the dipole attenuation contribution to high- $p_{T}$ hadron production in HIC is not sufficient and requires to include additionally more complicated and model dependent dynamics. It was demonstrated in [12, 13] that besides coherent effects a significant suppression of hadrons at forward rapidities, $x_{F} \gtrsim 0.1$, can be caused also by energy conservation constraints.

Since such additional nuclear suppression rises steeply with $x_{F}$ and/or $x_{T}$, it is convenient to introduce the variable $\xi=\sqrt{x_{F}^{2}+x_{T}^{2}}$, with $x_{F}=2 p_{L} / \sqrt{s}$ and $x_{T}=2 p_{T} / \sqrt{s}$, where $p_{L}$ and $p_{T}$ is the longitudinal and transverse component of the momentum of the produced particles in c.m. frame.

In the vicinity of the kinematic limit $\xi \rightarrow 1$ any hard reaction can be treated as a large rapidity gap (LRG) process where no particle is produced within rapidity interval $\Delta y=-\ln (1-\xi)$. The suppression factor as a survival probability for LRG was evaluated in [12], $S(\xi) \approx 1-\xi$. Then the parton distribution in the proton of the projectile nucleus $A$ can be expressed in terms of the nuclear thickness function $T_{B}(\vec{b})$ of the nucleus $B$ and the effective cross section $\sigma_{\text {eff }}=20 \mathrm{mb}$ [12],

$$
F_{i / p}^{(B)}\left(x_{1}, k_{1, T}^{2}, \vec{b}\right)=C F_{i / p}\left(x_{1}, k_{1, T}^{2}\right) \frac{\exp \left[-[1-S(\xi)] \sigma_{e f f} T_{B}(\vec{b})\right]-\exp \left[-\sigma_{e f f} T_{B}(\vec{b})\right]}{S(\xi)\left\{1-\exp \left[-\sigma_{e f f} T_{B}(\vec{b})\right]\right\},}
$$

where the normalization factor $C$ is fixed by the Gottfried sum rule.

Including energy conservation constraints one should modify also the expression (2.5) replacing $F_{i / A} \Rightarrow F_{i / A}^{(B)}(\vec{\tau})$ and $F_{j / B} \Rightarrow F_{j / B}^{(A)}(\vec{b}-\vec{\tau})$.
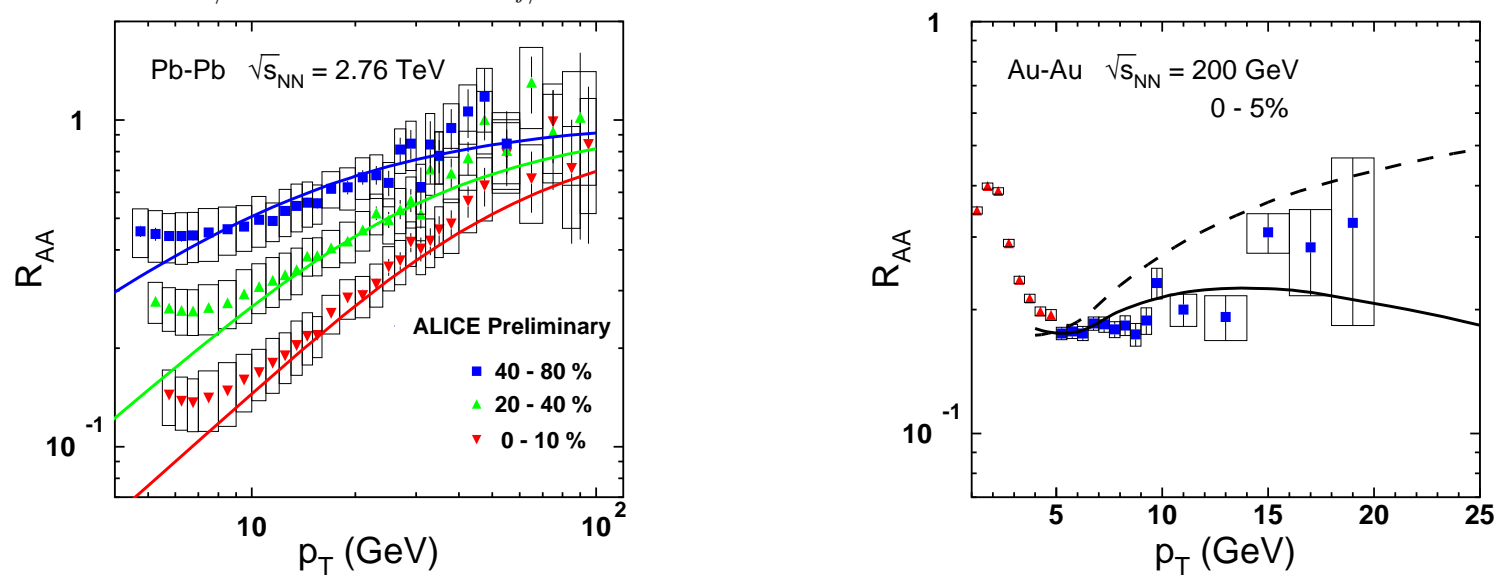

Figure 2: (Left) $R_{A A}\left(p_{T}\right)$ as function of $p_{T}$ for charged hadrons produced in lead-lead collisions at $\sqrt{s}=2.76 \mathrm{TeV}$ and at different centralities $0-10 \%, 20-40 \%$ and $40-80 \%$. Calculations with $\hat{q}_{0}=0.6 \mathrm{GeV}^{2} / \mathrm{fm}$ are compared with the ALICE data [1], 15]. (Right) $R_{A A}\left(p_{T}\right)$ as function of $p_{T}$ for neutral pions produced in central, $0-5 \%$, gold-gold collisions at $\sqrt{s}=200 \mathrm{GeV}$. Calculations with $\hat{q}_{0}=0.45 \mathrm{GeV}^{2} / \mathrm{fm}$ are compared with the PHENIX data [3] - triangles and [4] squares. The solid and dashed line corresponds to calculations including and excluding energy conservation constraints, respectively.

\section{RESULTS VS DATA}

Results of calculations with Eqs. (2.3)-(2.6) and (3.1) and with $\hat{q}_{0}=0.6 \mathrm{GeV}^{2} / \mathrm{fm}$ are shown

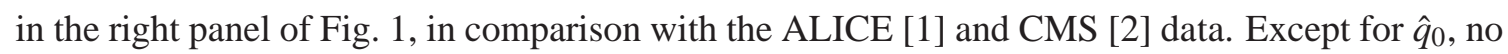
further adjustment was done, and agreement with data at large $p_{T}>7 \mathrm{GeV}$ is rather good. 
The left panel of Fig. 2 shows $p_{T}$ behavior of $R_{A A}$ at different centralities. Using the same value $\hat{q}_{0}=0.6 \mathrm{GeV}^{2} / \mathrm{fm}$ one can see again a good agreement of calculations with the ALICE data [1, 15] at large $p_{T}>7 \mathrm{GeV}$. The steep rise with $p_{T}$ of $R_{A A}$ is due to color transparency effects and is slightly slowing down since quarks with larger $l_{p}$ become more dominant.

In comparison with LHC a dominance of quarks with larger $l_{p}$ leads to a weaker suppression at RHIC. Such an expectation is confirmed by the PHENIX data [3, 包] as is shown in the right panel of Fig. 2. With the same description of the dipole propagation in a medium we obtained predictions depicted by the dashed line with adjusted value $\hat{q}_{0}=0.45 \mathrm{GeV}^{2} / \mathrm{fm}$. After inclusion of energy conservation constraints, Eq. (3.1), we obtained predictions presented by the solid line. This brings into a much better agreement with data at $p_{T}>5 \mathrm{GeV}$ exhibiting so rather flat $p_{T}$ dependence.
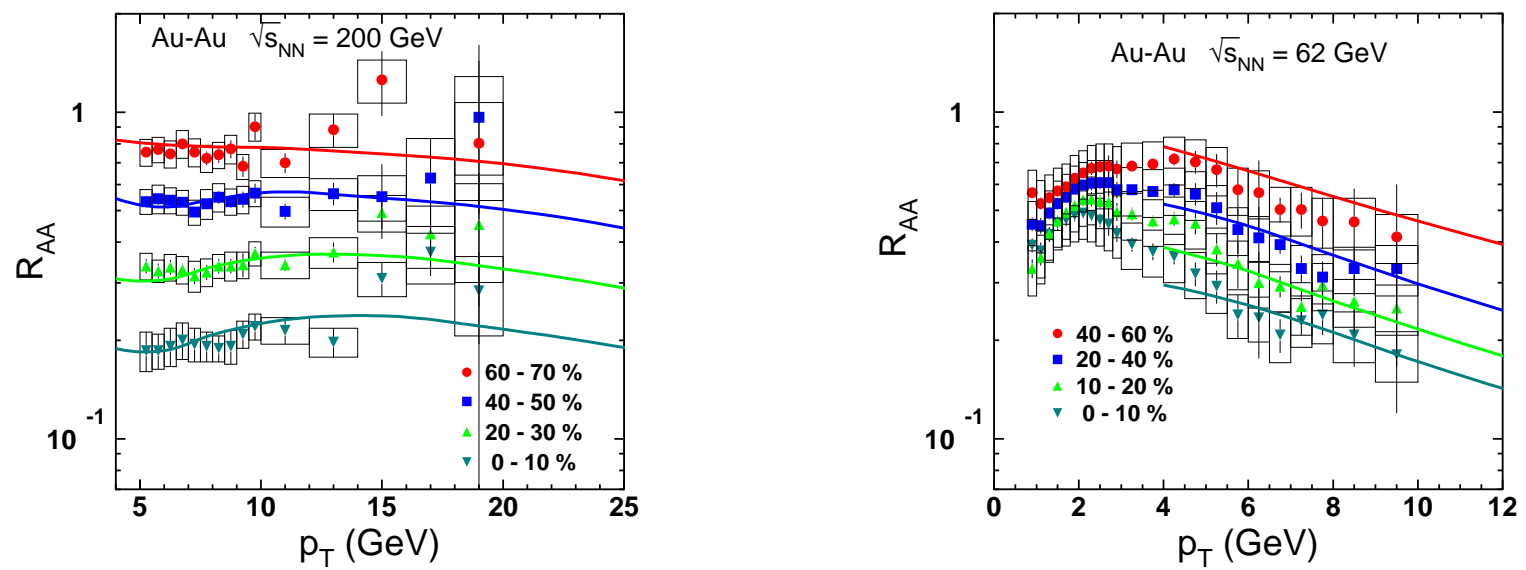

Figure 3: (Left) $R_{A A}\left(p_{T}\right)$ as function of $p_{T}$ for neutral pions produced in gold-gold collisions at $\sqrt{s}=200 \mathrm{GeV}$ and at different centralities $0-10 \%, 20-30 \%, 40-50 \%$ and $60-70 \%$. Calculations with $\hat{q}_{0}=0.45 \mathrm{GeV}^{2} / \mathrm{fm}$ are compared with the PHENIX data [17]. (Right) The same as the left panel but at lower c.m. energy $\sqrt{s}=62 \mathrm{GeV}$ and at different centralities $0-10 \%, 10-20 \%, 20-40 \%$ and $40-60 \%$. Calculations with $\hat{q}_{0}=0.3 \mathrm{GeV}^{2} / \mathrm{fm}$ are compared with the PHENIX data [1]].

Using the same adjusted value $\hat{q}_{0}=0.45 \mathrm{GeV}^{2} / \mathrm{fm}$ at $\sqrt{s}=200 \mathrm{GeV}$ we calculated $p_{T}$ behavior of $R_{A A}$ at different centralities and found a nice agreement with the PHENIX data [17] at $p_{T}>5 \mathrm{GeV}$ as is shown in the left panel of Fig. 国.

Another way to make effects related to energy conservation constraints stronger is to decrease the energy of a collison. Thus we can predict that a flat $p_{T}$ dependence of $R_{A A}$ at $\sqrt{s}=200 \mathrm{GeV}$ should be changed for a rise of nuclear suppression with $p_{T}$ at smaller $\sqrt{s}=62 \mathrm{GeV}$. Such an

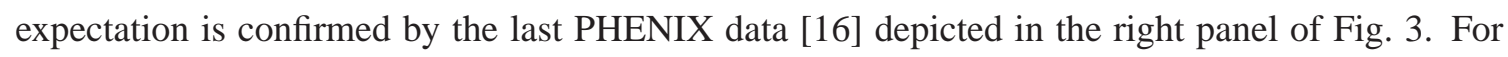
calculations we used $\hat{q}_{0}=0.30 \mathrm{GeV}^{2} / \mathrm{fm}$ adjusted from the central gold-gold collisions. One can see that agreement with data is rather good for $p_{T}>5 \mathrm{GeV}$.

\section{SUMMARY}

We applied a simple model for a dipole propagation in a medium using the standard convolution expression for description of high- $p_{T}$ hadron production in HIC. The dynamics of a strong nuclear suppression is based on the shortness of $l_{p}$ of a colorless dipole (pre-hadron) and on its devepment and propagation through a dense medium described by the survival probability leading to a steep rise of $R_{A A}$ with $p_{T}$ (color transparency effect). In comparison with LHC a dominance of quarks with larger $l_{p}$ leads to a weaker suppression at RHIC. In the RHIC kinematic region we 
introduced also an additional suppression factor related to the constraints on nuclear parton distributions imposed by the energy conservation. This suppression factor falling steeply with $p_{T}$ is irrelevant at LHC but causes rather flat $p_{T}$ dependence of $R_{A A}$ at RHIC c.m. energy $\sqrt{s}=200 \mathrm{GeV}$. At smaller $\sqrt{s}=62 \mathrm{GeV}$ this factor leads even to a rise of suppression with $p_{T}$. Calculations contain only medium density adjustment and we found the transport coefficients $\hat{q}_{0}=0.30 \mathrm{GeV}^{2} / \mathrm{fm}$ at $\sqrt{s}=62 \mathrm{GeV}, \hat{q}_{0}=0.45 \mathrm{GeV}^{2} / \mathrm{fm}$ at $\sqrt{s}=200 \mathrm{GeV}$ and $\hat{q}_{0}=0.60 \mathrm{GeV}^{2} / \mathrm{fm}$ at $\sqrt{s}=2.76 \mathrm{TeV}$. These calculations can be further improved using the rigorous quantum-mechanical approach based on the path integral technique for description of the $\bar{q} q$ dipole evolution.

This work was supported in part by Fondecyt (Chile) grants 1090291 and 1090236, by the Slovak Funding Agency, Grant 2/0092/10, by the Slovak Research and Developmnent Agency APVV-0050-11 and by Grants VZ MŠMT 6840770039 and LA 08015 (Ministry of Education of the Czech Republic).

\section{References}

[1] J. Otwinowski [for the ALICE Collaboration], J. Phys. G 38, 124112 (2011).

[2] Y.-J. Lee [for the CMS Collaboration], J. Phys. G 38, 124015 (2011). A. S. Yoon [for the CMS Collaboration], J. Phys. G 38, 124116 (2011).

[3] PHENIX Collaboration, S. S. Adler et al., Phys. Rev. Lett. 91, 072301 (2003), PHENIX Collaboration, A. Adare et al., Phys. Rev. Lett. 101, 232301 (2008).

[4] M. L. Purschke [for the PHENIX Collaboration], J. Phys. G 38, 124016 (2011).

[5] STAR Collaboration, J. Adams et al., Phys. Rev. Lett. 91, 072304 (2003).

[6] B.Z. Kopeliovich, J. Nemchik, E. Predazzi and A. Hayashigaki, Nucl. Phys. A 740, 211 (2004).

[7] B.Z. Kopeliovich and J. Nemchik, J. Phys. G 38, 043101 (2011).

[8] B.Z. Kopeliovich, H.J. Pirner, I.K. Potashnikova and I. Schmidt, Phys. Lett. B 662, 117 (2008).

[9] B.Z. Kopeliovich, I.K. Potashnikova and I. Schmidt, Phys. Rev. C 82, 024901 (2010).

[10] B.Z. Kopeliovich, I.K. Potashnikova and I. Schmidt, Phys. Rev. C 83, 021901 (2011).

[11] R.P. Feynman, R.D. Field and G.C. Fox, Phys. Rev. D 18, 3320 (1978).

[12] B.Z. Kopeliovich et al., Phys. Rev. C 72, 054606 (2005); Nucl. Phys. B 146, 171 (2005).

[13] J. Nemchik, V. Petracek, I.K. Potashnikova and M. Sumbera, Phys. Rev. C 78, 025213 (2008).

[14] X.F. Chen, C. Greiner, E. Wang, X.N. Wang and Z. Xu, Phys. Rev. C 81, 064908 (2010).

[15] H. Appelshauser [for the ALICE Collaboration], J. Phys. G 38, 124014 (2011).

[16] PHENIX Collaboration, preliminary data presented at URL: http : //www.phenix.bnl.gov/WWW/plots/show ${ }_{p}$ lot.php?editkey $=p 1118$

[17] Y. Aramaki [for the PHENIX Collaboration], presented at the Japanese Physical Society, Fall (Autumn), Kyusyu Institute of Technology, Kitakyushu, Japan, September 11-14, 2010.

[18] A.D. Martin, W.J. Stirling, R.S. Thorne and G. Watt, Eur. Phys. J. C 63, 189 (2009).

[19] K.J. Eskola, H. Paukkunen and C.A. Salgado, J. High Energy Phys. 04, 065 (2009).

[20] D. de Florian, R. Sassot and M. Stratmann, Phys. Rev. D 75, 114010 (2007); Phys. Rev. D 76, 074033 (2007).

[21] H.De Vries, C.W.De Jager and C.De Vries, Atomic Data and Nucl. Data Tables 36, 469 (1987). 